\title{
Analyzing Gene Expression Profiles with Preliminary Validations in Cardiac Hypertrophy Induced by Pressure- overload
}

\begin{tabular}{|c|c|}
\hline Journal: & Canadian Journal of Physiology and Pharmacology \\
\hline Manuscript ID & cjpp-2017-0585.R2 \\
\hline Manuscript Type: & Article \\
\hline Date Submitted by the Author: & 13-Dec-2017 \\
\hline Complete List of Authors: & $\begin{array}{l}\text { Gao, Jing; The First Hospital of China Medical University, Department of } \\
\text { Cardiovascular Ultrasound; The First Affiliated Hospital of Jinzhou Medical } \\
\text { University, Department of Ultrasonography } \\
\text { Li, Yuhong; The First Affiliated Hospital of Jinzhou Medical University, } \\
\text { Department of Ultrasonography } \\
\text { Wang, Tongmei; Jinzhou Medical University, Department of } \\
\text { Pathophysiology } \\
\text { Shi, Zhuo; Jinzhou Medical University, Department of Anatomy } \\
\text { Zhang, Yiqi; Jinzhou Medical University, Department of Pathophysiology } \\
\text { Liu, Shuang; The First Hospital of China Medical University, Department of } \\
\text { Cardiovascular Ultrasound } \\
\text { Wen, Pushuai; Jinzhou Medical University, Department of Pathophysiology } \\
\text { Ma, Chunyan; The First Hospital of China Medical University, Department of } \\
\text { Cardiovascular Ultrasound }\end{array}$ \\
\hline Keyword: & $\begin{array}{l}\text { Cardiac hypertrophy, Differentially expressed genes, Isoprenaline, } \\
\text { Functional enrichment analysis, Pathway enrichment analysis }\end{array}$ \\
\hline $\begin{array}{l}\text { Is the invited manuscript for } \\
\text { consideration in a Special } \\
\text { Issue?: }\end{array}$ & N/A \\
\hline
\end{tabular}


Analyzing Gene Expression Profiles with Preliminary Validations in Cardiac Hypertrophy Induced by Pressureoverload

Jing Gao ${ }^{1,2}$, Yuhong $\mathrm{Li}^{2}$, Tongmei Wang ${ }^{3}$, Zhuo Shi ${ }^{4}$, Yiqi Zhang ${ }^{3}$, Shuang Liu ${ }^{1}$, Pushuai Wen ${ }^{3^{*}}$, Chunyan Ma ${ }^{*}$

${ }^{1}$ Department of Cardiovascular Ultrasound, The First Hospital of China Medical University, Shenyang 110001, China;

${ }^{2}$ Department of Ultrasonography, The First Affiliated Hospital of Jinzhou Medical University, Jinzhou 121001, China;

${ }^{3}$ Department of Pathophysiology, Jinzhou Medical University, Jinzhou 121001, China;

${ }^{4}$ Department of Anatomy, Jinzhou Medical University, Jinzhou 121001, China.

\section{* Corresponding author:}

Pushuai Wen, Ph.D.

No.40, Section III, Songpo Road, Linghe District, Jinzhou, Liaoning Province, P.R. China.

Tel: +86-(0)416-4673331

E-mail: wenpushuai@163.com

Chunyan Ma, M. D.

No.77 Puhe Road, Shenyang North New Area, Shenyang, Liaoning Province, P.R. China

Tel: +86-(0)24-83282114

E-mail: mcy_echo@163.com 


\begin{abstract}
The aim of this study was to identify the key genes involved in the cardiac hypertrophy $(\mathrm{CH})$ induced by pressure overload. mRNA microarray dataset GSE5500 and GSE18801 were downloaded from GEO database, and differentially expressed genes (DEGs) were screened using Limma package; then, functional and pathway enrichment analysis were performed for common DEGs using DAVID database. Furthermore, the top DEGs were further validated using qPCR in the hypertrophic heart tissue induced by Isoprenaline (ISO). A total of 113 common DEGs with absolute fold change $>0.5$, including 60 significantly up-regulated DEGs and 53 down-regulated DEGs were obtained. GO term enrichment analysis suggested that common up-regulated DEG mainly enriched in neutrophil chemotaxis, extracellular fibril organization and cell proliferation, and the common down-regulated genes were significantly enriched in ion transport, endoplasmic reticulum and dendritic spine. KEGG pathway analysis found that the common DEGs were mainly enriched in ECM-receptor interaction, phagosome, and focal adhesion. Additionally, the expression of Mfap4, Ltbp2, Aspn, Serpina3n, and Cnksr1 were up-regulated in the model of cardiac hypertrophy, while the expression of Anp32a was down-regulated. The current study identified the key deregulated genes and pathways involved in the $\mathrm{CH}$, which could shed new light to understand the mechanism of $\mathrm{CH}$.
\end{abstract}

Keywords Cardiac hypertrophy; Differentially expressed genes; Pathway enrichment analysis; Isoprenaline; Functional enrichment analysis. 


\section{Introduction}

The updated data of heart disease and stroke statistics (2016 Edition) from the American Heart Association (AHA) showed that cardiovascular disease (CVD) is still the leading cause of death worldwide (Writing Group et al. 2016). Due to the increasing number of people living with diseases and the aging of the population, the family and society must face and solve this serious problem of prevention and treatment of cardiovascular disease.

Myocardial hypertrophy is a disorder of changing structures and functions of the heart in a variety of CVD conditions. Among them, pressure overload induced by hypertension is one of the most common causes for myocardial hypertrophy (Honsho et al. 2009; Rossi 1998). Myocardial hypertrophy is characterized by an increased myocardial cell volume, sarcomere hyperplasia, and accompanied with the fetal gene reactivation related to myocardial hypertrophy (Oparil 1985; Taegtmeyer et al. 2010). In the early stage of the pathological process of CVD, cardiac hypertrophy is an adaptive response of the heart, which is beneficial to improve the heart function and reduce the ventricular wall tension. However, the long-term and persistent pathological cardiac hypertrophy often leads to cardiac dilatation, diastolic dysfunction and a series of decompensatory reactions, eventually leading to heart failure and sudden death (Benjamin et al. 2017). It has been confirmed that the pathological cardiac hypertrophy results from the pathological stimuli involving many signaling pathway, such as beta adrenergic receptors, PI3K/AKT, and $\mathrm{Ca}^{2+} /$ calmodulin dependent protein kinase II pathway (Liu et al. 2007; Vidal et al. 2012; Zhang and Brown, 2004). Nevertheless, the mechanism of cardiac hypertrophy has not been fully elucidated yet.

The gene expression chip, an effective technique for revealing the relationship between disease and genes, and providing the valuable clues for the pathogenesis of the diseases, has been widely used in a variety of disease research fields, including cardiovascular disease (Henriksen and Kotelevtsev, 2002; Tarca et al. 2006).

In the present study, we re-analyzed the gene expression profiles of GSE5500 and GSE18801 to explore the molecular mechanisms of cardiac hypertrophy induced by pressure-overload (Bisping et al., 2006; Galindo et al., 2009). The correlation between DEGs and cardiac hypertrophy was examined through a comprehensive bioinformatics analysis including biological process annotation and biological pathway enrichment analysis. Finally, we confirmed the expressions of DEGs in the myocardial tissue of mice with hypertension-induced cardiac hypertrophy, which may provide the potential molecular targets involved in the cardiac hypertrophy. 


\section{Materials and methods}

\section{Expression profile microarray}

Data was downloaded from the Gene Expression Omnibus (GEO), a public repository for data storage (http://www.ncbi.nlm.nih. gov/geo/) (Edgar et al. 2002). The microarray dataset GSE5500 and GSE18801, in which the RNA extracted from the ventricles of mice with cardiac hypertrophy induced by pressure-overload was analyzed using the GPL1261 platform (Affymetrix Mouse Genome 4302.0 arrays), were downloaded from GEO database. GSE5500 dataset included 4 sham-operated samples and 6 TAC (transverse aortic constriction) samples of mice, which have same genetic background (FVB/N mice), sex (male), and similar body weight (22.5 25.5 g). And, the microarray data of GSE18801 had 3 sham-operated and 3 ISO infusion samples, which resulted from eight-week old male C57BL/6 mice (Bisping et al. 2006; Galindo et al. 2009) (Table 1).

\section{Identification of DEGs}

Samples were divided into two groups: sham-operated samples and cardiac hypertrophy samples. DEGs between the sham-operated samples and cardiac hypertrophy samples were identified, respectively. Firstly, the background correction and normalization of the microarray data were performed in the R language (Troyanskaya et al., 2001), and DEGs were subsequently identified using a Limma package in R (Wettenhall and Smyth, 2004). The significance of DEGs was calculated by t-test and was represented by $P$ value. $P<0.05$ and absolute $\log 2$ fold change $(\mathrm{FC})>0.5$ were chosen as the cut-off criteria.

\section{GO terms and KEGG pathway enrichment analysis}

The Database for Annotation, Visualization and Integrated Discovery (DAVID, http://david.abcc.ncifcrf.gov/) is an online program that provides a comprehensive set of functional annotation tools for researchers to understand the biological meaning behind plenty of genes(Huang da et al. 2009). Gene ontology (GO) and Kyoto Encyclopedia of Genes and Genomes (KEGG) pathway enrichment analysis were performed for identified DEGs using DAVID database (version 6.7, National Institutes of Health). P value 0.05 was used as a cut-off for significance.

\section{Animal and experimental protocol}

The animal experimental protocols were approved by the Animal Care and Use Committee of Jinzhou Medical University. All experimental procedures used in this study were conducted in accordance with the guidelines of the Canadian Council on Animal Care. 
Male Kunming mice weighing 24-28 g aged 4 weeks were acclimatized in the laboratory for 1 week before experiment. The animal model of cardiac hypertrophy was induced via continuous infusion of isoprenaline $(5 \mathrm{mg} / \mathrm{kg} / \mathrm{d}$, ) or saline by implanting an osmotic minipump (Alzet model 1002, Cupertino, USA), as described previously (Ma et al. 2011). The mice were randomly divided into two experimental groups with 6 mice in each group: saline infusion group and isoprenaline infusion group.

\section{Volume pressure recording}

Systolic blood pressure (SBP), diastolic blood pressure (DBP) and mean arterial blood pressure (MBP) were measured by volume pressure recording of the tail using the CODA noninvasive blood pressure system (Kent Scientific Corp, Torrington, USA) in conscious mice following $\geqq 3$ training periods, as described previously (Daugherty et al. 2009).

\section{Histological measurement}

After euthanization, the body and heart weights of mice were recorded. Additionally, the hearts were fixed, sectioned (5 $\mu \mathrm{m})$, and counterstained with hematoxyline-eosin (HE). Mean cross-sectional area (CSA) (>100 cells/section) was measured from transverse sections, using Image J software (Liu et al. 2009).

\section{Echocardiographic measurement}

Echocardiographic studies were performed using an ultrasonographic system (GE Healthcare, Milwaukee, USA) equipped with a $8-\mathrm{MHz} 10 \mathrm{~S}$ probe. After anesthetization with ketamine/xylazine combination, M-mode pictures captured from the left parasternal long axis view were recorded to measure end-diastolic interventricular septum thickness (IVSd), left ventricular posterior wall thickness at end-diastole (LVPWd), left ventricular internal diameters at end-diastole (LVIDd), and fractional shortening (FS) were calculated with the established standard equation. Echocardiographic measurements were performed in triplicate and then were averaged (Jiang et al. 2014).

\section{Quantitative RT-PCR}

The mRNA expressions of DEGs in cardiac hypertrophy were measured by qPCR, with normalization to GAPDH expression. Briefly, total RNA was extracted from myocardial tissue using Trizol (Invitrogen, Thermo Fisher Scientific Inc., Waltham, USA). Genomic DNA was removed from RNA samples by digestion with DNaseI, according to the manufacturer's instructions. First strand cDNA was synthesized from $2 \mu \mathrm{g}$ of total RNA using the oligo (dT) primers and Moloney Murine Leukemia Virus Reverse Transcriptase (M-MLV RT, Madison, USA) according to the manufacturer's instructions. Real-Time PCR was used to quantify transcript levels and performed on the ABI StepOne real-time PCR using Absolute Blue SYBR green PCR mix (Thermo Fisher Scientific Inc., Waltham, USA). The following primers were 
used: Anp32a (5'-CAGGGGACCTGGAAGTATTGG-3' and 5'-TTCAGGTTGGTCACCTCACAG-3'), Mfap4 (5'ATGGACGGCAGTGTGAAT-3' and 5'-CTTCTTGTGGCGTGTCAG-3') (Wang et al. 2015), Ltbp2 (5'-AAACCCCT CAGCGACCCGCGGCTGC-3' and 5'-TGCTTCTGTGAGGACCGGGTGCTCT-3') (Fujikawa et al., 2017), Aspn (5'CCATTtGGGTGCCAATGTtACT-3' and 5'-TGGGTGAATCTTTGTtAGCTTGT-3'), Serpina3n (5'-CAATGTCTG CGAAACTGTACC-3' and 5'-TTtGGGGTtGGCTATCTTGGC-3'), Cnksr1 (5'-TCCTCACCTGC TAAGCCAATC-3' and 5'-GACTGAGCGTCAAAGGAGGTA-3'), and D17H6S56E (5'-CCCAGACGGGATCAAG ACTC-3' and 5'-GGG TTCATGGGGCATAGGAC-3'); we used a 2-step amplification $\left(40\right.$ cycles of $95^{\circ} \mathrm{C}, 15 \mathrm{~s} ; 65^{\circ} \mathrm{C}, 30 \mathrm{~s} ; 72^{\circ} \mathrm{C}, 30 \mathrm{~s}$; followed by melting temperature determination stage) and fold enrichment was calculated by the $2^{-\Delta \Delta C T}$ method.

\section{Statistical analysis}

All grouped data are presented as mean \pm SEM (Standard Error of Mean). To determine statistical significance, results were compared by using one-way analysis of variance (ANOVA) followed by Tukey's HSD test. $P<0.05$ at $95 \%$ confidence level was considered significant. 


\section{Results}

\section{Identification of DEGs}

Gene expression datasets GSE5500 and GSE18801 were downloaded from GEO datasets. After data normalization (Figure 1a and 1b) and differential expression analysis, a total of 429 and 2349 DEGs were identified from GSE5500 and GSE18801 datasets, respectively (Figure 1c and d). Among them, 278 up-regulated genes and 151 down-regulated genes in GSE5500 dataset, and 866 up-regulated genes and 1483 down-regulated genes in GSE18801 dataset, were identified. In addition, the 113 common DEGs were obtained, comprising of 63 co-up-regulated genes and 50 co-down-regulated gene (Figure 1 e and f, Supplementary Table S1 and S2). Furthermore, 17 genes were identified as the most significantly deregulated genes in $\mathrm{CH}$ when the cutoff of absolute fold change was increased more than 1 . The heat maps for these 17 genes are displayed in Figure 2.

\section{Biological classification and pathway enrichment analysis of common DEGs}

To gain insights into the GO categories of common DEGs from cardiac hypertrophy induced by pressure-overload, we uploaded all common DEGs to the DAVID database. As to the biological process, the up-regulated DEGs were significantly enriched in biological processes (BP), including neutrophil chemotaxis, extracellular fibril organization, positive regulation of inflammatory response, positive regulation of calcium ion import, and ossification; the downregulated genes were significantly enriched in biological processes, including ion transport, potassium ion transmembrane transport, potassium ion transport, and regulation of ion transmembrane transport; for cell component (CC), the up-regulated DEGs were significantly enriched in cell proliferation, cell differentiaion, negative regulation of protein kinase activity, cell response to tumor necrosis factor, extracellular region; the down-regulated DEGs were significantly enriched in endoplasmic reticulum and dendritic spine; in addition, molecular function (MF) analysis also displayed that the up-regulated DEGs were significantly enriched in collagen trimer, basement membrane, cell surface, neuronmuscular junction, and heparin binding; the down-regulated DEGs were significantly enriched in mRNA 3'-UTR binding, potassium channel activity, calcium ion binding, and voltage-gated ion channel activity (Figure 3a and 3b) .

After KEGG pathway enrichment analysis, the common DEGs were mainly enriched in nine KEGG pathways, such as ECM-receptor interaction, phagosome, focal adhesion, amoebiasis, tuberculosis, PI3K-Akt signaling pathway, small cell lung cancer p53 signaling pathway, protein digestion and absorption (Table 2). Thus, these significantly enriched GO terms and pathways could help us to further understand the roles of these DEGs involved in the occurrence and development of $\mathrm{CH}$ induced by by pressure-overload. 


\section{The establishment of cardiac hypertrophy model in mice}

To make sure the model of cardiac hypertrophy was induced by pressure overload, we detected the blood pressure for 2 weeks. The data indicated that the ISO treatment elevated SBP, DBP and MBP in mice significantly, as shown in Figure 4a. Subsequently, we detected the hypertrophic responses of these mice in response to ISO stimulation. The ISO treated mice exhibited a significant hypertrophic phenotype, such as the increased heart weight/body weight (HW/BW) and increased cross-sectional area (Table 3 and Figure 4b). To investigate the cardiac hypertrophy and cardiac function further, we performed the echocardiographic examination. ISO treatment increased the left ventricular hypertrophy, judged by increased LVPWd and IVSd, with the enlargement of the left ventricular chamber judged by decreased the LVIDd (Table 3 and Figure 4c); Percent fractional shortening (\%FS) reflecting the systolic function of LV did not differ between sham and ISO treated mouse (Table 3). Collectively, these results suggested that we established the models of concentric hypertrophy in the compensatory stage, which was characterized as the elevated after-load, increased relative wall thickness and conserved contractile function.

\section{Validation of expression levels of the most significantly common DEGs in animal model of cardiac hypertrophy}

Next, we detected the mRNA expression level of the selected most significantly common DEGs by qPCR method in the mouse model of ISO-induced cardiac hypertrophy, excluding the 10 published desregulated genes, such as Postn, Myh7, Lgals3, Mfap5, Timp1, Spp1, Cthrc1, Ctgf, Clip and Mt2 (Ahmed et al., 2004; Galindo et al., 2009; Heymans et al., 2005; k et al., 2017; Miyazaki et al., 2006; van Berlo, Elrod, Aronow, Pu, \& Molkentin, 2011; J. J. Wang et al., 2016; Xue et al., 2010). Compared with the sham group, the significantly reduced expression levels of Anp32a and the significantly high expression of Mfap4, Ltbp2, Aspn, Serpina3n, and Cnksr1 were validated in the animal model with cardiac hypertrophy, while D17H6S56E-5 was not significantly differentially expressed, which was not consistent with bioinformatics investigation (Figure 5).

\section{Discussion}

Pressure-overload induced myocardial hypertrophy is an important risk factor for heart failure, sudden death, arrhythmia and many other cardiovascular diseases. Currently, it's believed that the following factors are involved in the occurrence and development of $\mathrm{CH}$, such as angiotensin, catecholamines, endothelin-1, transforming growth factor-1, and IL-1 (Crowley et al., 2006; Dobaczewski et al. 2011; Irukayama-Tomobe et al. 2004; Orn et al. 2012; Scheuer 1999). In addition, it has been confirmed that the pathological cardiac hypertrophy resulting from the pathological stimuli involved many signaling pathway, such as beta adrenergic receptors, $\mathrm{PI} 3 \mathrm{~K} / \mathrm{AKT}, \mathrm{Ca}^{2+} /$ calmodulin dependent protein kinase II, 
MAPKs, AMPK, NFKB pathways (Dyck and Lopaschuk 2006; Li et al. 2004; Liu et al. 2007; Streicher et al. 2010; Vidal et al. 2012; Zhang and Brown 2004). Although many studies have been devoted to explore the pathogenesis of $\mathrm{CH}$, the mechanisms of $\mathrm{CH}$ development and progression have not been elucidated fully yet.

Gene expression profiling studies based on the microarray technology have been developing rapidly and has been widely used to reveal the general genetic alterations in progression of diseases, which enables the understanding of the pathogenesis and identification of novel regulators and pathways involved the occurrence and development of $\mathrm{CH}$ (Wang et al. 2016; Westenbrink et al. 2015).

In the present study, two microarray datasets were obtained to identify the common DEGs between hearts with $\mathrm{CH}$ induced by pressure-overload and control samples, perform GO and pathway analysis for DEGs. The result of GO and pathway analysis for the common DEGs indicated that these common DEGs between two datasets were mainly enriched in ECM-receptor interaction, phagosome, focal adhesion, and PI3K-Akt signaling pathway, which may be closely related to $\mathrm{CH}$, which was in accordance with previous study. For example, ECM (extracellular matrix) including type I and type III fibrillar collagens play very important roles in the remodeling in heart disease (Berk et al. 2007); furthermore, phenylephrine-induced cardiac hypertrophy in vitro was more pronounced in CTSL (Cathepsin-L, a key member of the lysosomal protease family)- deficient neonatal cardiomyocytes than in in controls, accompanied by a significant accumulation of autophagosomes, which indicated that facilitation of autophagy and proteasomal protein processing is essential for the preserved cardiac function(Sun et al. 2013); and, when the focal adhesion kinase (FAK) is activated in cardiomyocytes, the mice developed the eccentric cardiac hypertrophy upon stimulation with angiotensin II or pressure overload by transverse aortic constriction (Peng et al. 2006); in addition, FAK signaling is required for the PE-induced hypertrophy in isolated neonatal cardiomyocytes (Taylor et al. 2000); several investigators have demonstrated that the PI3K-Akt pathway regulates cardiomyocyte size, survival, angiogenesis, and inflammation in both physiological and pathological cardiac hypertrophy (Aoyagi and Matsui 2011).

Besides, in the present study, we established the mouse model of concentric hypertrophy in compensatory stage induced by ISO and validated the expression of DEGs with the most significant changes in the hypertrophic myocardium tissues, which is helpful to understand the signaling pathway involved in the compensatory cardiac hypertrophy induced by pressure overload. A total of 113 common DEGs were screened, consisting of 63 up-regulated genes and 50 downregulated genes. Among them, we identified the expression of the most significantly common DEGs in animal model 
with $\mathrm{CH}$, including Aspn, Mfap4, Ltbp2, Anp32a, Cnksr1, and Serpina3n, which may be correlated with the occurrence and development of $\mathrm{CH}$ closely.

The previous study demonstrated that distinct signal transduction pathways were activated in different types of cardiac hypertrophy (Kehat et al. 2011; Miyamoto et al. 2004; Sopontammarak et al. 2005). Adult myocytes from hearts of mice with genetic deletion of all ERK1/2 showed preferential eccentric growth, conversely, activated Mek1( mitogen-activated protein kinase kinase 1) promoted constitutive ERK1/2 signaling and increased myocyte thickness, which implied the activation of ERK1/2 signaling pathway can regulate the the balance between eccentric and concentric cardiac growth (Kehat et al. 2011). In the present study, some of validated DEGs in our ISO-induced CH model are associated with ERK1/2 pathways, including Cnksr1 and Anp32a.

Firstly, Cnksr1 (connector enhancer of kinase suppressor of Ras 1), as a regulator and binding partner of the Kinase Suppressor of Ras-1 (KSR1), can directly modulate response to small molecule inhibition of the MAPK pathway (Quadri et al. 2017). Furthermore, Cnksrl functions as a scaffold in several signal cascades and acts on proliferation, differentiation and apoptosis (Fritz and Radziwill 2005). Cnksr1 can interact with the angiotensin II type-2 receptor (AT2R), indicating it may play a role in the AT2R mediated signaling pathway, which have been implicated in many cardiovascular conditions, such as hypertension, atherosclerosis, coronary heart disease, restenosis, and heart failure (Fritz and Radziwill, 2005; Lemarie and Schiffrin 2010). Additionally, Xue J et al., found that compared with transgenic mice expressing wild-type $\mathrm{Na}^{+} / \mathrm{H}^{+}$exchanger isoform 1 (NHE1), the mice expressing the activated NHE1 showed a significant cardiac hypertrophy phenotype, and the expression of Cnksr1 in the myocardium of wild-type NHE1expressing transgenic mice is down-regulated significantly, which indicates that the level of NHE1 activity is important for the occurrence of cardiac hypertrophy (Xue et al. 2010). Taken together, these data support the hypothesis the upregulated Cnksr1 and related signaling pathway may act as a key role associated with compensatory concentric hypertrophy.

Secondly, Anp32a (acidic nuclear phosphoprotein 32 family member A), as a tumor suppressor, has been implicated in a broad array of physiological processes, including cell differentiation, apoptotic cell death, cell proliferation, and mammalian development via the actions of phosphatase inhibition, chromatin regulation, caspase activation, and intracellular transport ((Hoffarth et al. 2008; Reilly et al. 2011). Moreover, Anp32a .has been identified as an inhibitor of protein phosphatase $2 \mathrm{~A}$, leading to changes in the ERK signaling pathway, which implied down-regulated Anp32 may 
associate with concentric growth of the heart (Habrukowich et al. 2010; Kehat et al. 2011). However, there is no report on the effects of Anp32a on cardiac hypertrophy so far, which requires the further study. Ltbp2 (latent transforming growth factor beta binding protein 2) belonging to the family of fibrillin/Ltbp glycoproteins, is another up-regulated protein in this study. The structures of repetitive EGF-like domains and 8-cystein repeats in Ltbp family are believed to mediate protein-protein interactions, such as fibrillin- 1 and transforming growth factor- $\beta$ (TGF- $\beta$ ) (Isogai et al. 2003); Zhang group found that Fibulin-2 deficiency attenuates angiotensin-induced cardiac hypertrophy by reducing transforming growth factor- $\beta$ signalling (Zhang et al. 2014); in addition, LT Ltbps can facilitate TGF- $\beta$ secretion and directing its ECM localization, which play very important roles in the pathogenesis of $\mathrm{CH}$ (Horiguchi et al. 2012; Sterner-Kock et al. 2002).

Serpina3n, serpin family A member 3n, inhibits neutrophil cathepsin $G$ and mast-cell chymase, which convert angiotensin I to II, resulting in vasoconstriction, fluid retention, and increased blood pressure (Dhahbi et al. 2006). Serpina3 was increased in dyssynchrony heart failure (DHF) but then reversed towards the normal level after resynchronization therapy (Yang et al. 2015). Microarray analysis in failing myocardium discovered that Serpina3 gene is involved in mitochondrial dysfunction and oxidative phosphorylation (Xue et al. 2010). These evidences suggested that serpina3n may be involved in the pathogenesis of $\mathrm{CH}$.

Aspn (asporin), encodes a cartilage extracellular protein that is member of the small leucine-rich proteoglycan family, which is highly expressed in osteoarthritis cartilage tissue, inhibiting TGF- $\beta$ signaling-mediated synthesis of cartilage specific extracellular matrix components, such as type II collagen and proteglycan in chondrocytes (Kaliakatsos et al. 2006). Similar with the Anp32a, there is also no relevant study about the effects of Aspn on cardiac hypertrophy so far.

Mfap4, microfibril Associated Protein 4, an extracellular matrix protein which is involved in cell adhesion or intercellular interactions. It has been reported that serum Mfap4 acts as a biomarker of stable atherosclerotic disease (Wulf-Johansson et al. 2013). Similarly, serum Mfap4 varies in groups of patients with various cardiovascular conditions (Wulf-Johansson et al. 2013). Furthermore, in dyssynchronous heart failure, the secreted Mfap4 exhibited upregulation (Yang et al. 2015). In the model of chronic isoproterenol application to induce cardiac hypertrophy, Myoglobin knockout mice $\left(\right.$ myo $^{-/}$) displayed conserved cardiac function and no signs of left ventricular dilatation, while Mfap4 expression is up-regulated in myo ${ }^{-/}$hearts after isoproterenol stimulation (Molojavyi et al. 2010). Thus, these results further confirm the usefulness of our findings of this present study. 
However, there are some limitations of our study. One limitation is that the microarray data are not generated by ourselves but from GEO database. The second limitation of the study was the relatively small sample size. Therefore, our results should be followed up in the further experimental studies based on a larger sample size.

In summary, the present study intended on identifying DEGs and biological pathways between hearts with $\mathrm{CH}$ induced by pressure-overload and control samples by data mining, bioinformatics analysis and biological identification, which may play an important role in the development of cardiac hypertrophy induced by pressure-overload, further providing more information for studies on pathogenesis and screening of potential biomarkers of cardiac hypertrophy.

\section{Acknowledgements}

This work was supported by the National Natural Science Foundation of China (grant No. 81501959), Doctoral Scientific

Research Starting Fundation of Liaoning Province (grant No. 2015010911-301), Technological Project of Liaoning Province (Grant No. 20170540392).

\section{Conflict of interest}

The authors declare that there are no competing interests associated with the manuscript. 


\section{References}

Ahmed, M.S., Oie, E., Vinge, L. E., Yndestad, A., Oystein Andersen, G., Andersson, Y., et al. 2004. Connective tissue growth factor--a novel mediator of angiotensin II-stimulated cardiac fibroblast activation in heart failure in rats. J. Mol. Cell Cardiol. 36(3): 393-404. doi: 10.1016/j.yjmcc.2003.12.004.

Aoyagi, T., and Matsui, T. 2011. Phosphoinositide-3 kinase signaling in cardiac hypertrophy and heart failure. Curr. Pharm. Des. 17(18): 1818-1824.

Benjamin, E. J., Blaha, M. J., Chiuve, S. E., Cushman, M., Das, S. R., Deo, R., et al. 2017. Heart Disease and Stroke Statistics-2017 Update: A Report From the American Heart Association. Circulation. 135(10): e146-e603. doi: 10.1161/CIR.0000000000000485.

Berk, B. C., Fujiwara, K., and Lehoux, S. 2007. ECM remodeling in hypertensive heart disease. J. Clin. Invest. 117(3): 568-575. doi: 10.1172/JCI31044.

Bisping, E., Ikeda, S., Kong, S.W., Tarnavski, O., Bodyak, N., McMullen, J. R., et al. 2006. Gata4 is required for maintenance of postnatal cardiac function and protection from pressure overload-induced heart failure. Proc. Natl. Acad. Sci. U S A. 103(39): 14471-14476. doi:10.1073/pnas.0602543103

Crowley, S. D., Gurley, S. B., Herrera, M. J., Ruiz, P., Griffiths, R., Kumar, A. P., et al. 2006. Angiotensin II causes hypertension and cardiac hypertrophy through its receptors in the kidney. Proc. Natl. Acad. Sci. U S A. 103(47): 17985-17990. doi:10.1073/pnas.0605545103.

Daugherty, A., Rateri, D., Hong, L., and Balakrishnan, A. 2009. Measuring blood pressure in mice using volume pressure recording, a tail-cuff method. J. Vis. Exp. (27): pii: 1291. doi:10.3791/1291.

Dhahbi, J. M., Tsuchiya, T., Kim, H. J., Mote, P. L., and Spindler, S. R. 2006. Gene expression and physiologic responses of the heart to the initiation and withdrawal of caloric restriction. J. Gerontol. A Biol. Sci. Med. Sci. 61(3): 218-231.

Dobaczewski, M., Chen, W., and Frangogiannis, N. G. 2011. Transforming growth factor (TGF)-beta signaling in cardiac remodeling. J. Mol. Cell Cardiol. 51(4): 600-606. doi: 10.1016/j.yjmcc.2010.10.033.

Dyck, J. R., and Lopaschuk, G. D. 2006. AMPK alterations in cardiac physiology and pathology: enemy or ally? J. Physiol. 574 (Pt 1): 95-112. doi:10.1113/jphysiol.2006.109389.

Edgar, R., Domrachev, M., and Lash, A. E. 2002. Gene Expression Omnibus: NCBI gene expression and hybridization array data repository. Nucleic Acids Res. 30(1): 207-210.

Fritz, R. D., and Radziwill, G. 2005. The scaffold protein CNK1 interacts with the angiotensin II type 2 receptor. Biochem. Biophys. Res. Commun. 338(4): 1906-1912. doi:10.1016/j.bbrc.2005.10.168.

Fujikawa, Y., Yoshida, H., Inoue, T., Ohbayashi, T., Noda, K., von Melchner, H., et al. 2017. Latent TGF-beta binding protein 2 and 4 have essential overlapping functions in microfibril development. Sci. Rep. 7: 43714. doi: $10.1038 /$ srep43714.

Galindo, C. L., Skinner, M. A., Errami, M., Olson, L. D., Watson, D. A., Li, J., et al. 2009. Transcriptional profile of isoproterenol-induced cardiomyopathy and comparison to exercise-induced cardiac hypertrophy and human cardiac failure. BMC Physiol. 9: 23. doi:10.1186/1472-6793-9-23.

Habrukowich, C., Han, D. K., Le, A., Rezaul, K., Pan, W., Ghosh, M., et al. 2010. Sphingosine interaction with acidic leucine-rich nuclear phosphoprotein-32A (ANP32A) regulates PP2A activity and cyclooxygenase (COX)-2 expression in human endothelial cells. J. Biol. Chem. 285(35): 26825-26831. doi: 10.1074/jbc.M110.147058.

Henriksen, P. A., and Kotelevtsev, Y. 2002. Application of gene expression profiling to cardiovascular disease. Cardiovasc. Res. 54(1): 16-24.

Heymans, S., Schroen, B., Vermeersch, P., Milting, H., Gao, F., Kassner, A., et al. 2005. Increased cardiac expression of tissue inhibitor of metalloproteinase-1 and tissue inhibitor of metalloproteinase-2 is related to cardiac fibrosis and dysfunction in the chronic pressure-overloaded human heart. Circulation. 112(8): 1136-1144. doi: 10.1161/ CIRCULATIONAHA.104.516963. 
Hoffarth, S., Zitzer, A., Wiewrodt, R., Hahnel, P. S., Beyer, V., Kreft, A., et al. 2008. pp32/PHAPI determines the apoptosis response of non-small-cell lung cancer. Cell Death Differ. 15(1): 161-170. doi:10.1038/sj.cdd.4402256.

Honsho, S., Nishikawa, S., Amano, K., Zen, K., Adachi, Y., Kishita, E., et al. 2009. Pressure-mediated hypertrophy and mechanical stretch induces IL-1 release and subsequent IGF-1 generation to maintain compensative hypertrophy by affecting Akt and JNK pathways. Circ. Res. 105(11): 1149-1158. doi: 10.1161/CIRCRESAHA.109.208199.

Horiguchi, M., Ota, M., and Rifkin, D. B. 2012. Matrix control of transforming growth factor-beta function. J. Biochem. 152(4): 321-329.doi: $10.1093 / \mathrm{jb} / \mathrm{mvs} 089$.

Huang da, W., Sherman, B. T., and Lempicki, R. A. 2009. Systematic and integrative analysis of large gene lists using DAVID bioinformatics resources. Nat. Protoc. 4(1): 44-57. doi:10.1038/nprot.2008.211.

ILAR (Institute of Laboratory Animal Resources). 1996. Guide for the Care and Use of Laboratory Animals. NIH Publication No. 85-23 (revised 1996). National Academy Press, Washington, D.C.

Irukayama-Tomobe, Y., Miyauchi, T., Sakai, S., Kasuya, Y., Ogata, T., Takanashi, M., et al. 2004. Endothelin-1-induced cardiac hypertrophy is inhibited by activation of peroxisome proliferator-activated receptor-alpha partly via blockade of c-Jun NH2-terminal kinase pathway. Circulation. 109(7): 904-910. doi:10.1161/01.CIR. 0000112596. 06954. 00 .

Isogai, Z., Ono, R. N., Ushiro, S., Keene, D. R., Chen, Y., Mazzieri, R., et al. 2003. Latent transforming growth factor beta-binding protein 1 interacts with fibrillin and is a microfibril-associated protein. J. Biol. Chem. 278(4): 27502757. doi:10.1074/jbc.M209256200.

Jiang, D. S., Wei, X., Zhang, X. F., Liu, Y., Zhang, Y., Chen, K., et al. 2014. IRF8 suppresses pathological cardiac remodelling by inhibiting calcineurin signalling. Nat. Commun, 5: 3303. doi:10.1038/ncomms4303.

Shindo, k., Asakura, M., Min, K. D., Ito, S., Fu, H. Y., Yamazaki, S., et al. 2017. Cartilage Intermediate Layer Protein 1 Suppresses TGF- $\beta$ Signaling in Cardiac Fibroblasts. Int. J. Gerontol. 11(2): 67-74.

Kaliakatsos, M., Tzetis, M., Kanavakis, E., Fytili, P., Chouliaras, G., Karachalios, T., et al. 2006. Asporin and knee osteoarthritis in patients of Greek origin. Osteoarthritis Cartilage. 14(6): 609-611.doi: 10.1016/j.joca.2005.10.012.

Kehat, I., Davis, J., Tiburcy, M., Accornero, F., Saba-El-Leil, M. K., Maillet, M., et al. 2011. Extracellular signalregulated kinases 1 and 2 regulate the balance between eccentric and concentric cardiac growth. Circ. Res. 108 (2): 176-183. doi: 10.1161/CIRCRESAHA.110.231514.

Lemarie, C. A., and Schiffrin, E. L. 2010. The angiotensin II type 2 receptor in cardiovascular disease. J. Renin Angiotensin Aldosterone Syst. 11(1): 19-31. doi: 10.1177/1470320309347785.

Li, Y., Ha, T., Gao, X., Kelley, J., Williams, D. L., Browder, I. W., et al. 2004. NF-kappaB activation is required for the development of cardiac hypertrophy in vivo. Am. J. Physiol. Heart Circ. Physiol. 287(4): H1712-1720. doi: 10.1152/ajpheart.00124.2004.

Liu, L., Zhao, X., Pierre, S. V., and Askari, A. 2007. Association of PI3K-Akt signaling pathway with digitalis-induced hypertrophy of cardiac myocytes. Am. J. Physiol. Cell Physiol. 293(5): C1489-1497. doi: 10.1152/ajpcell.00158. 2007.

Liu, W., Zi, M., Jin, J., Prehar, S., Oceandy, D., Kimura, T. E., et al. 2009. Cardiac-specific deletion of mkk4 reveals its role in pathological hypertrophic remodeling but not in physiological cardiac growth. Circ. Res. 104(7): 905-914.doi: 10.1161/CIRCRESAHA.108.188292.

Ma, X., Song, Y., Chen, C., Fu, Y., Shen, Q., Li, Z., et al. 2011. Distinct actions of intermittent and sustained betaadrenoceptor stimulation on cardiac remodeling. Sci. China Life Sci. 54(6): 493-501. doi: 10.1007/s11427-0114183-9

Miyamoto, T., Takeishi, Y., Takahashi, H., Shishido, T., Arimoto, T., Tomoike, H., et al. 2004. Activation of distinct signal transduction pathways in hypertrophied hearts by pressure and volume overload. Basic Res. Cardiol. 99(5): 328-337. doi; 10.1007/s00395-004-0482-7.

Miyazaki, H., Oka, N., Koga, A., Ohmura, H., Ueda, T., and Imaizumi, T. 2006. Comparison of gene expression profiling in pressure and volume overload-induced myocardial hypertrophies in rats. Hypertens. Res. 29(12): 10291045.doi: 10.1291/hypres.29.1029. 
Molojavyi, A., Lindecke, A., Raupach, A., Moellendorf, S., Kohrer, K., and Godecke, A. 2010. Myoglobin-deficient mice activate a distinct cardiac gene expression program in response to isoproterenol-induced hypertrophy. Physiol. Genomics. 41(2): 137-145.doi: 10.1152/physiolgenomics.90297.2008.

Oparil, S. (1985). Pathogenesis of ventricular hypertrophy. J. Am. Coll. Cardiol. 5(6 Suppl): 57B-65B.

Orn, S., Ueland, T., Manhenke, C., Sandanger, O., Godang, K., Yndestad, A., et al. 2012. Increased interleukin-1beta levels are associated with left ventricular hypertrophy and remodelling following acute ST segment elevation myocardial infarction treated by primary percutaneous coronary intervention. J. Intern. Med. 272(3): 267-276.doi: 10.1111/j.1365-2796.2012.02517.x.

Peng, X., Kraus, M. S., Wei, H., Shen, T. L., Pariaut, R., Alcaraz, A., et al. 2006. Inactivation of focal adhesion kinase in cardiomyocytes promotes eccentric cardiac hypertrophy and fibrosis in mice. J. Clin. Invest. 116(1): 217-227.doi: 10.1172/JCI24497.

Quadri, H. S., Aiken, T. J., Allgaeuer, M., Moravec, R., Altekruse, S., Hussain, S. P., et al. 2017. Expression of the scaffold connector enhancer of kinase suppressor of Ras 1 (CNKSR1) is correlated with clinical outcome in pancreatic cancer. BMC Cancer. 17(1): 495. doi: 10.1186/s12885-017-3481-4.

Reilly, P. T., Afzal, S., Gorrini, C., Lui, K., Bukhman, Y. V., Wakeham, A., et al. 2011. Acidic nuclear phosphoprotein $32 \mathrm{kDa}$ (ANP32)B-deficient mouse reveals a hierarchy of ANP32 importance in mammalian development. Proc. Natl. Acad. Sci U S A. 108(25): 10243-10248.doi: 10.1073/pnas.1106211108.

Rossi, M. A. 1998. Pathologic fibrosis and connective tissue matrix in left ventricular hypertrophy due to chronic arterial hypertension in humans. J. Hypertens. 16(7): 1031-1041.

Scheuer, J. 1999. Catecholamines in cardiac hypertrophy. Am. J. Cardiol. 83(12A): 70H-74H.

Sopontammarak, S., Aliharoob, A., Ocampo, C., Arcilla, R. A., Gupta, M. P., and Gupta, M. 2005. Mitogen-activated protein kinases (p38 and c-Jun NH2-terminal kinase) are differentially regulated during cardiac volume and pressure overload hypertrophy. Cell Biochem. Biophys. 43(1): 61-76. doi: 10.1385/CBB:43:1:061.

Sterner-Kock, A., Thorey, I. S., Koli, K., Wempe, F., Otte, J., Bangsow, T., et al. 2002. Disruption of the gene encoding the latent transforming growth factor-beta binding protein 4 (LTBP-4) causes abnormal lung development, cardiomyopathy, and colorectal cancer. Genes Dev. 16(17): 2264-2273. doi:10.1101/gad.229102.

Streicher, J. M., Ren, S., Herschman, H., and Wang, Y. 2010. MAPK-activated protein kinase-2 in cardiac hypertrophy and cyclooxygenase-2 regulation in heart. Circ. Res. 106(8): 1434-1443.doi: 10.1161/CIRCRESAHA.109.213199.

Sun, M., Ouzounian, M., de Couto, G., Chen, M., Yan, R., Fukuoka, M., et al. 2013. Cathepsin-L ameliorates cardiac hypertrophy through activation of the autophagy-lysosomal dependent protein processing pathways. J. Am. Heart Assoc. 2(2): e000191.doi: 10.1161/JAHA.113.000191.

Taegtmeyer, H., Sen, S., and Vela, D. 2010. Return to the fetal gene program: a suggested metabolic link to gene expression in the heart. Ann. N. Y. Acad. Sci. 1188: 191-198.doi: 10.1111/j.1749-6632.2009.05100.x.

Tarca, A. L., Romero, R., and Draghici, S. 2006. Analysis of microarray experiments of gene expression profiling. Am. J. Obstet. Gynecol. 195(2): 373-388. doi:10.1016/j.ajog.2006.07.001.

Taylor, J. M., Rovin, J. D., and Parsons, J. T. 2000. A role for focal adhesion kinase in phenylephrine-induced hypertrophy of rat ventricular cardiomyocytes. J. Biol. Chem. 275(25): 19250-19257.doi: 10.1074/jbc.M909099199.

Troyanskaya, O., Cantor, M., Sherlock, G., Brown, P., Hastie, T., Tibshirani, R., et al. 2001. Missing value estimation methods for DNA microarrays. Bioinformatics. 17(6): 520-525.

van Berlo, J. H., Elrod, J. W., Aronow, B. J., Pu, W. T., and Molkentin, J. D. 2011. Serine 105 phosphorylation of transcription factor GATA4 is necessary for stress-induced cardiac hypertrophy in vivo. Proc. Natl. Acad. Sci U S A. 108(30): 12331-12336.doi: 10.1073/pnas.1104499108.

Vidal, M., Wieland, T., Lohse, M. J., and Lorenz, K. 2012. beta-Adrenergic receptor stimulation causes cardiac hypertrophy via a Gbetagamma/Erk-dependent pathway. Cardiovasc. Res. 96(2): 255-264. doi:10.1093/cvr/cvs249. 
Wang, J. J., Rau, C., Avetisyan, R., Ren, S., Romay, M. C., Stolin, G., et al. 2016. Genetic Dissection of Cardiac Remodeling in an Isoproterenol-Induced Heart Failure Mouse Model. PLoS Genet. 12(7): e1006038.doi: 10.1371/journal.pgen.1006038.

Wang, S., He, Q., Ma, D., Xue, Y., and Liu, F. 2015. Irf4 Regulates the Choice between T Lymphoid-Primed Progenitor and Myeloid Lineage Fates during Embryogenesis. Dev. Cell. 34(6): 621-631. doi:10.1016/j.devcel.2015.07.011.

Westenbrink, B. D., Ling, H., Divakaruni, A. S., Gray, C. B., Zambon, A. C., Dalton, N. D., et al. 2015. Mitochondrial reprogramming induced by CaMKIIdelta mediates hypertrophy decompensation. Circ. Res. 116(5): e28-39.doi: 10.1161/CIRCRESAHA.116.304682

Wettenhall, J. M., and Smyth, G. K. 2004. limmaGUI: a graphical user interface for linear modeling of microarray data. Bioinformatics. 20(18): 3705-3706. doi:10.1093/bioinformatics/bth449.

Writing Group, M., Mozaffarian, D., Benjamin, E. J., Go, A. S., Arnett, D. K., Blaha, M. J., et al. 2016. Heart Disease and Stroke Statistics-2016 Update: A Report From the American Heart Association. Circulation.133(4): e38-360. doi: 10.1161/CIR.0000000000000350.

Wulf-Johansson, H., Lock Johansson, S., Schlosser, A., Trommelholt Holm, A., Rasmussen, L. M., Mickley, H., et al. 2013. Localization of microfibrillar-associated protein 4 (MFAP4) in human tissues: clinical evaluation of serum MFAP4 and its association with various cardiovascular conditions. PLoS One. 8(12): e82243. doi: 10.1371/ journal.pone. 0082243 .

Xue, J., Mraiche, F., Zhou, D., Karmazyn, M., Oka, T., Fliegel, L., et al. 2010. Elevated myocardial $\mathrm{Na}^{+} / \mathrm{H}^{+}$exchanger isoform 1 activity elicits gene expression that leads to cardiac hypertrophy. Physiol. Genomics. 42(3): 374-383. doi:10.1152/physiolgenomics.00064.2010.

Yang, S., Chen, L., Sun, S., Shah, P., Yang, W., Zhang, B., et al. 2015. Glycoproteins identified from heart failure and treatment models. Proteomics. 15(2-3): 567-579. doi:10.1002/pmic.201400151.

Zhang, H., Wu, J., Dong, H., Khan, S. A., Chu, M. L., and Tsuda, T. 2014. Fibulin-2 deficiency attenuates angiotensin IIinduced cardiac hypertrophy by reducing transforming growth factor-beta signalling. Clin. Sci (Lond). 126(4): 275288. doi:10.1042/CS20120636.

Zhang, T., and Brown, J. H. 2004. Role of $\mathrm{Ca}^{2+} /$ calmodulin-dependent protein kinase II in cardiac hypertrophy and heart failure. Cardiovasc. Res. 63(3): 476-486. doi: 10.1016/j.cardiores.2004.04.026. 
Table 1. GEO Datasets used for the analysis.

\begin{tabular}{cccc}
\hline Datasets & Methods of CH models & Samples & Platform \\
\hline GSE5500 & TAC induced-Pressure Overload & 4 sham-operated and 6 TAC samples & GPL1261, Affymetrix Mouse \\
Genome 430 2.0 Array \\
$\begin{array}{c}\text { GSE1880 } \\
1\end{array}$ & ISO-induced Pressure Overload & 3 sham-operated and 3 ISO infusion samples & $\begin{array}{c}\text { GPL1261, Affymetrix Mouse } \\
\text { Genome 430 2.0 Array }\end{array}$ \\
\hline
\end{tabular}

Note: Transverse aortic constriction 
Table 2. The enriched KEGG pathway of common DEGs.

\begin{tabular}{|c|c|c|c|c|c|}
\hline Category & Term & Description & Count & P Value & Genes \\
\hline KEGG & mmu04512 & ECM-receptor interaction & 5 & $1.66 \mathrm{E}-04$ & $\begin{array}{l}\text { COL4A2, COL4A1, COL5A2, } \\
\text { THBS4, SPP1 }\end{array}$ \\
\hline KEGG & mmu04115 & p53 signaling pathway & 4 & $1.25 \mathrm{E}-03$ & $\begin{array}{l}\text { CDK1, CCNB2, SERPINE1, } \\
\text { GADD45G }\end{array}$ \\
\hline KEGG & mmu04145 & Phagosome & 5 & $2.18 \mathrm{E}-03$ & $\begin{array}{l}\text { MRC1, CTSS, CD14, THBS4, } \\
\text { FCGR3 }\end{array}$ \\
\hline KEGG & mmu04510 & Focal adhesion & 5 & $4.08 \mathrm{E}-03$ & $\begin{array}{l}\text { COL4A2, COL4A1, COL5A2, } \\
\text { THBS4, SPP1 }\end{array}$ \\
\hline KEGG & mmu05146 & Amoebiasis & 4 & $6.12 \mathrm{E}-03$ & $\begin{array}{l}\text { COL4A2, COL4A1, COL5A2, } \\
\text { CD14 }\end{array}$ \\
\hline KEGG & mmu05152 & Tuberculosis & 4 & $1.85 \mathrm{E}-02$ & MRC1, CTSS, CD14, FCGR3 \\
\hline KEGG & mmu04151 & PI3K-Akt signaling pathway & 5 & $2.49 \mathrm{E}-02$ & $\begin{array}{l}\text { COL4A2, COL4A1, COL5A2, } \\
\text { THBS4, SPP1 }\end{array}$ \\
\hline KEGG & mmu05222 & Small cell lung cancer & 3 & $2.98 \mathrm{E}-02$ & COL4A2, COL4A1, CKS2 \\
\hline KEGG & mmu04974 & Protein digestion and absorption & 3 & $3.25 \mathrm{E}-02$ & COL4A2, COL4A1, COL5A2 \\
\hline
\end{tabular}

Notes: Count, the number of DEGs; KEGG, Kyoto Encyclopedia of Genes and Genomes 
Table 3. Assessment of cardiac hypertrophy induced by ISO.

\begin{tabular}{rcc}
\hline Parameters & $\begin{array}{c}\text { Saline infusion group } \\
(\mathbf{n}=\mathbf{6})\end{array}$ & $\begin{array}{c}\text { ISO infusion group } \\
(\mathbf{n}=\mathbf{6})\end{array}$ \\
\hline Heart weight, HW, mg & $116.58 \pm 1.49$ & $140.32 \pm 2.14^{* *}$ \\
Body weight, BW, g & $28.71 \pm 0.20$ & $27.95 \pm 0.29$ \\
HW/BW & $4.06 \pm 0.06$ & $4.91 \pm 0.08^{* *}$ \\
CSA, $\mu m^{2}$ & $195.78 \pm 10.42$ & $240.12 \pm 9.56^{* *}$ \\
LVIDd & $3.20 \pm 0.11$ & $2.21 \pm 0.34^{* *}$ \\
IVSd & $0.63 \pm 0.06$ & $0.78 \pm 0.05 *$ \\
LVPWd & $0.42 \pm 0.08$ & $0.83 \pm 0.11^{* *}$ \\
FS, $\%$ & $36.97 \pm 0.48$ & $37.77 \pm 0.43$ \\
\hline
\end{tabular}

Note: $* * \mathrm{P}<0.01$ vs sham, ${ }^{*} \mathrm{P}<0.05$ vs sham.

\section{Figure legends}

Fig. 1. Normalization of gene expression data, volcano plot of DEGs and venn diagram of DEGs in mRNA expression profiling datasets. ( $a$ and $b$ ) left panel, gene expression profiles before normalization; right panel, gene expression profiles after normalization; white boxes represent sham-operated samples, while gray boxes stand for cardiac hypertrophy samples. (c and d) Volcano plot of DEGs in sham-operated samples and cardiac hypertrophy samples in GSE5500 and GSE18801 datasets, respectively. DEGs were selected by $\mathrm{P}<0.05 \& \mid \log 2$ (fold change) $\mid>0.5$. The $\mathrm{x}$-axis shows the fold change in gene expression between sham-operated samples and cardiac hypertrophy samples, and the yaxis shows the statistical significance of the differences. Splashes represent different genes. Red splashes mean genes without significant different expression. Blue splashes means significantly expressed genes. (e and f) Venn diagrams illustrates the number of up and down-regulated genes in both datasets, respectively.

Fig. 2. The heatmaps of the top-regulated common DEGs in 2 datasets. Microarray data heatmap illustrating the different expression patterns of 17 top genes with $\mathrm{P}<0.05$ and absolute fold changes $>0.5$ in both datasets. Each row represents one of 17 genes, and each column represents each sample. The column on the left clusters the samples based on similarity in fold change among the samples. Green color represents a higher $\log 2$ fold change while red illustrates a lower $\log 2$ fold change. The scale on the right matches colours to $\log 2$ fold change values. 
Fig. 3. GO classification of common DEGs in the listed categories. a and b: GO enrichment analysis results of upregulated or down-regulated common DEGs with $\mathrm{P}<0.05$ and absolute fold changes $>0.5$, respectively. The top $\mathrm{x}$ axis represents the number of genes in the marked category; the bottom $\mathrm{x}$ axis indicated the minus Log10 (P) of categories. Only functional categories with $\mathrm{P}<0.05$ are shown.

Fig. 4. Established animal model of cardiac hypertrophy in mouse. (a) Effect of ISO on systolic blood pressure (SBP), diastolic blood pressure (DBP) and mean blood pressure(MBP). (b) Representative photomicrographs of the heart and myocytes (I and II). Cardiomyocytes cross-sectional area significantly increased after ISO treatment (III and IV); Bar: 20 $\mu \mathrm{m}$. (c) Representative examples of M-mode echocardiograms of hearts from sham control or ISO treatment infusion mice, respectively. ISO increased LVSd and LVPWd wall dimensions and decreased the LVIDd compared with the sham control mice.

Fig. 5. Real-time quantitative PCR validation of expression levels of the selected most significantly common DEGs. mRNA levels of ANP32a, Mfap4, Ltbp2, Aspn, Serpina3n, Cnksr1 and D17H6S56E-5 in heart tissues of mice in sham operated group and ISO treated group. Values shown (Y axis represents relative expression units) are normalized to GAPDH; **: $\mathrm{P}<0.01, * * *: \mathrm{P}<0.001, \mathrm{NS}:$ not significant. 

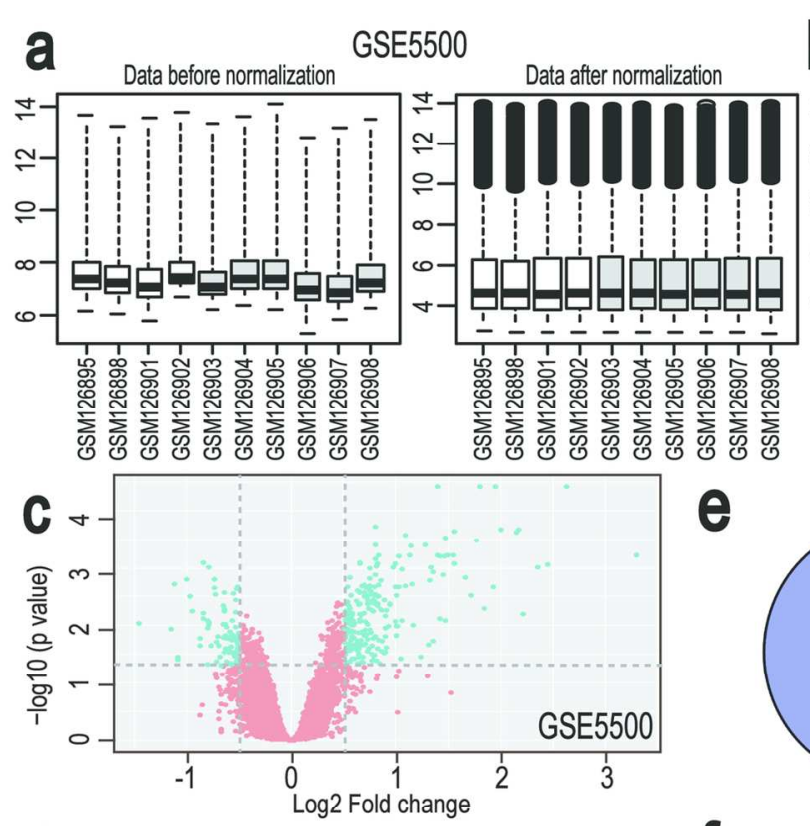

e

b. GSE18801 Data before normalization Data after normalization
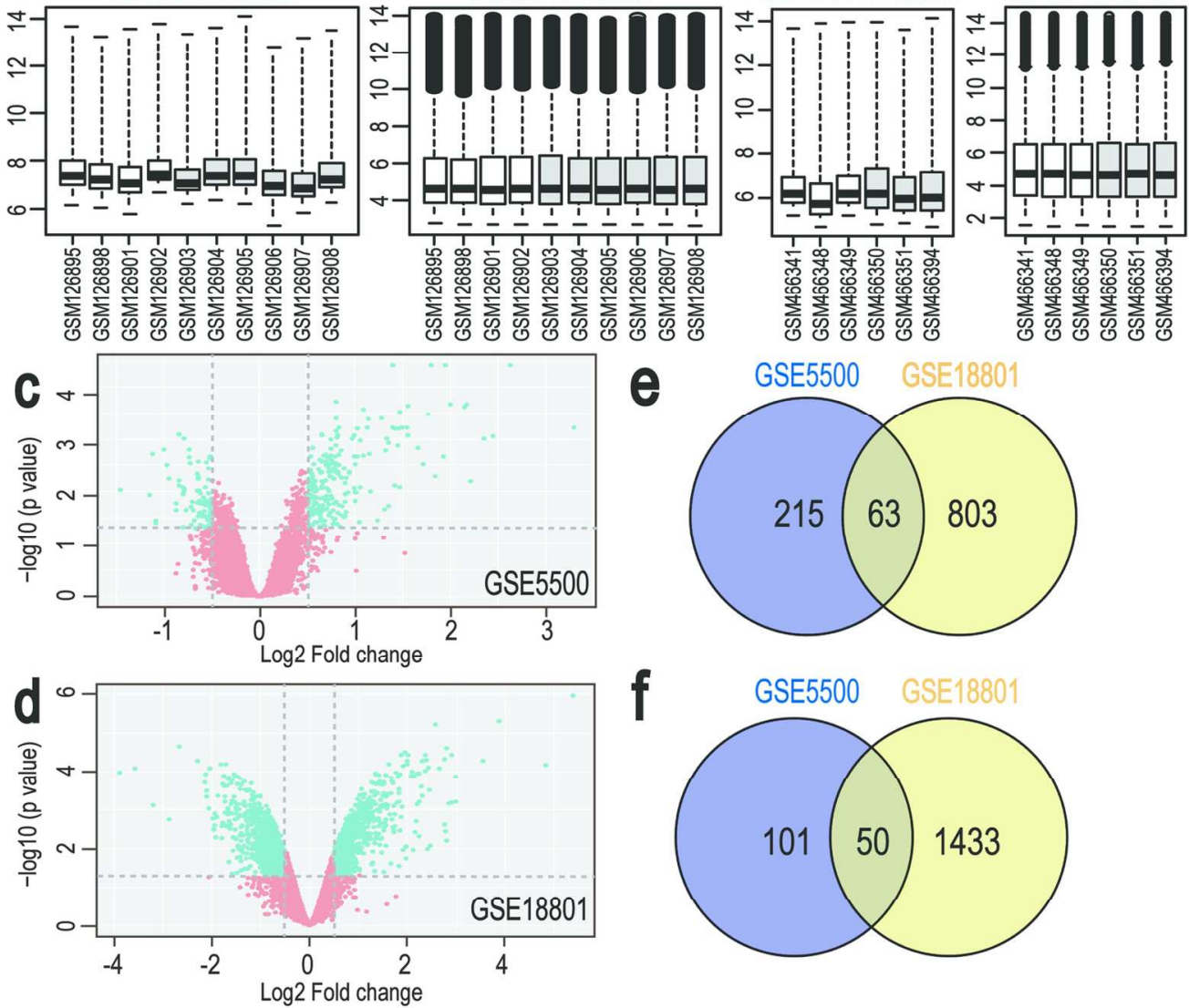

Fig. 1. Normalization of gene expression data, volcano plot of DEGs and venn diagram of DEGs in mRNA expression profiling datasets. ( $a$ and $b$ ) left panel, gene expression profiles before normalization; right panel, gene expression profiles after normalization; white boxes represent sham-operated samples, while gray boxes stand for cardiac hypertrophy samples. (c and d) Volcano plot of DEGs in sham-operated samples and cardiac hypertrophy samples in GSE5500 and GSE18801 datasets, respectively. DEGs were selected by $P<0.05 \& \mid \log 2$ (fold change) $\mid>0.5$. The $x$-axis shows the fold change in gene expression between sham-operated samples and cardiac hypertrophy samples, and the $y$-axis shows the statistical significance of the differences. Splashes represent different genes. Red splashes mean genes without significant different expression. Blue splashes means significantly expressed genes. (e and f) Venn diagrams illustrates the number of up and down-regulated genes in both datasets, respectively.

$139 \times 128 \mathrm{~mm}(300 \times 300 \mathrm{DPI})$ 


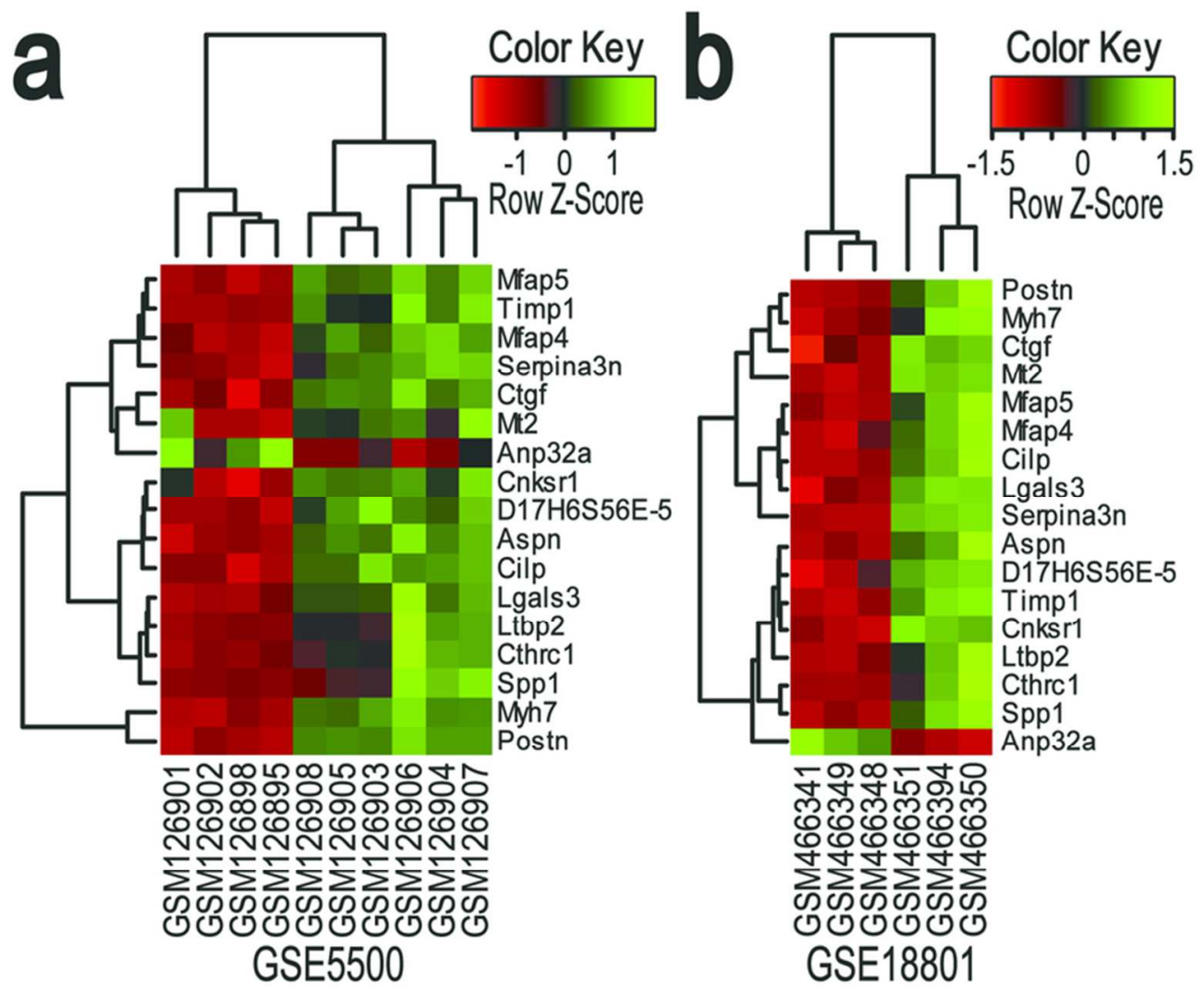

Fig. 2. The heatmaps of the top-regulated common DEGs in 2 datasets. Microarray data heatmap illustrating the different expression patterns of 17 top genes with $\mathrm{P}<0.05$ and absolute fold changes $>0.5$ in both datasets. Each row represents one of 17 genes, and each column represents each sample. The column on the left clusters the samples based on similarity in fold change among the samples. Green color represents a higher $\log 2$ fold change while red illustrates a lower log2 fold change. The scale on the right matches colours to log2 fold change values.

$73 \times 60 \mathrm{~mm}(300 \times 300$ DPI $)$ 


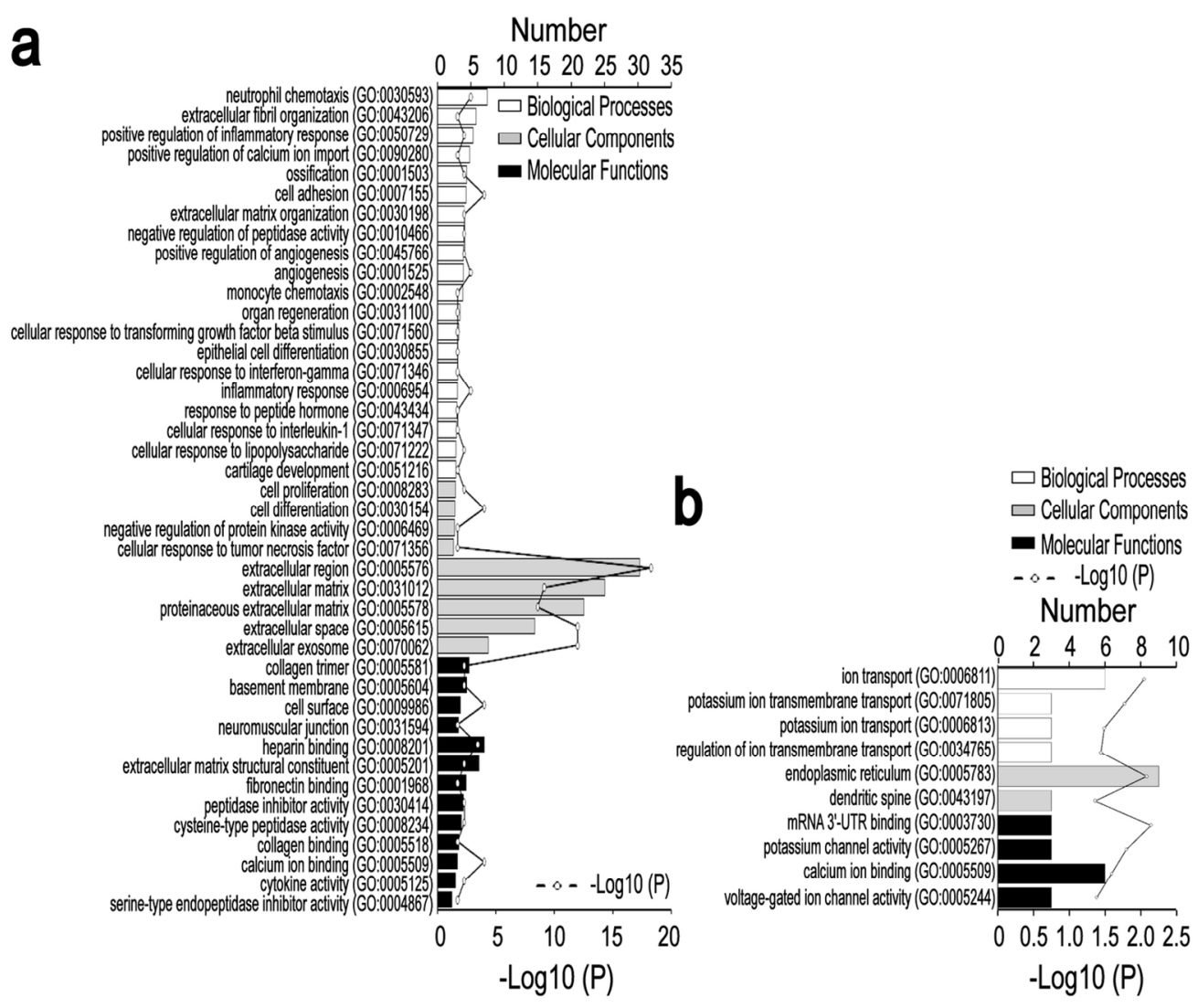

Fig. 3. GO classification of common DEGs in the listed categories. a and b: GO enrichment analysis results of up-regulated or down-regulated common DEGs with $\mathrm{P}<0.05$ and absolute fold changes $>0.5$, respectively. The top $\mathrm{x}$ axis represents the number of genes in the marked category; the bottom $\mathrm{x}$ axis indicated the minus Log10 $(P)$ of categories. Only functional categories with $\mathrm{P}<0.05$ are shown.

$127 \times 105 \mathrm{~mm}(300 \times 300 \mathrm{DPI})$ 

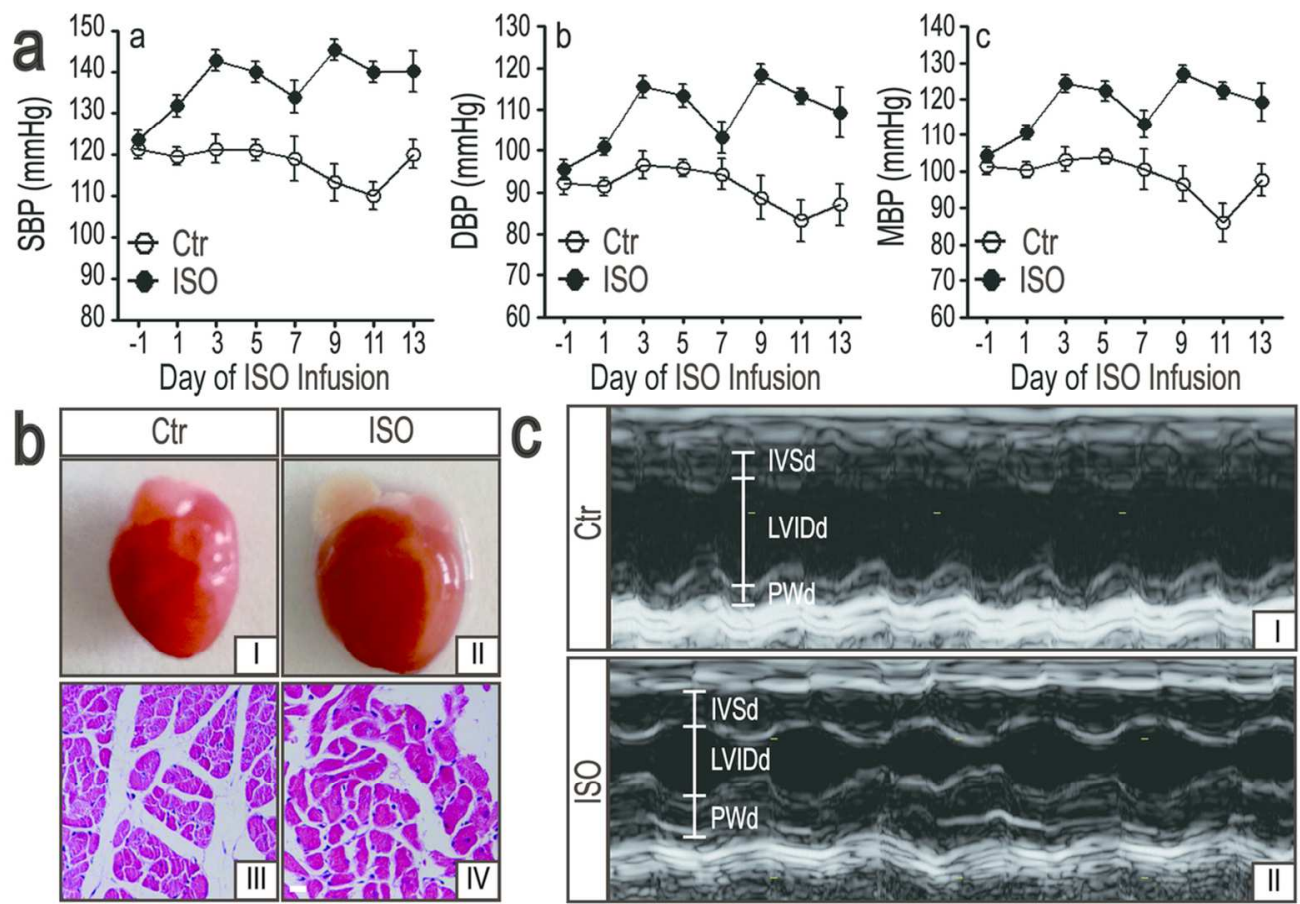

Fig. 4. Established animal model of cardiac hypertrophy in mouse. (a) Effect of ISO on systolic blood pressure (SBP), diastolic blood pressure (DBP) and mean blood pressure(MBP). (b) Representative photomicrographs of the heart and myocytes (I and II). Cardiomyocytes cross-sectional area significantly increased after ISO treatment (III and IV); Bar: $20 \mu \mathrm{m}$. (c) Representative examples of M-mode echocardiograms of hearts from sham control or ISO treatment infusion mice, respectively. ISO increased LVSd and LVPWd wall dimensions and decreased the LVIDd compared with the sham control mice.

$107 \times 75 \mathrm{~mm}(300 \times 300 \mathrm{DPI})$ 


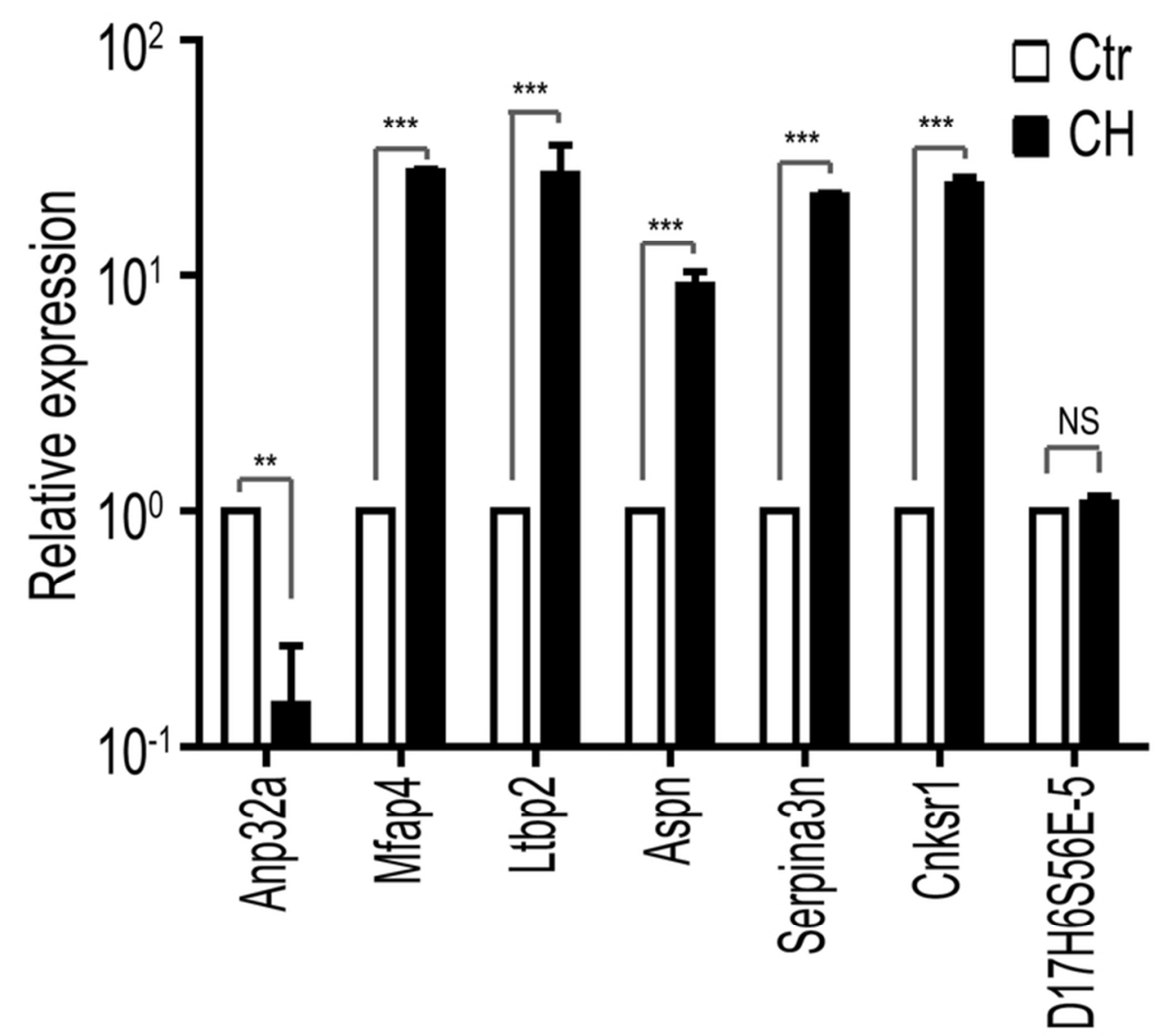

Fig.5. Real-time quantitative PCR validation of expression levels of the selected most significantly common DEGs. mRNA levels of ANP32a, Mfap4, Ltbp2, Aspn, Serpina3n, Cnksr1 and D17H6S56E-5 in heart tissues of mice in sham operated group and ISO treated group. Values shown ( $Y$ axis represents relative expression units) are normalized to GAPDH; **: $\mathrm{P}<0.01, * * *: \mathrm{P}<0.001, \mathrm{NS}$ : not significant.

$67 \times 59 \mathrm{~mm}(300 \times 300$ DPI $)$ 